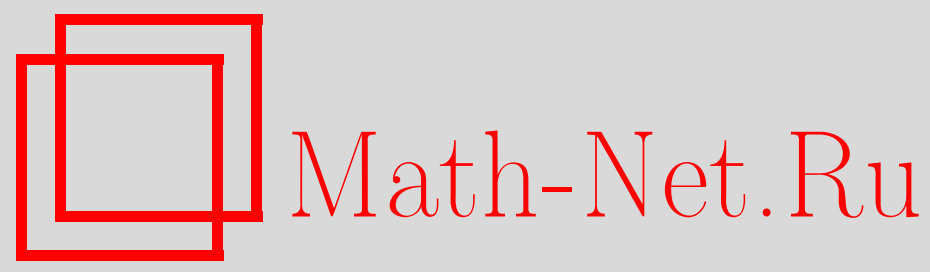

И. И. Котова, Описание систем корней с данной группой Вейля, Матем. заметки, 1998, том 64, выпуск 3, 397-402

DOI: https://doi.org/10.4213/mzm1409

Использование Общероссийского математического портала Math-Net.Ru подразумевает, что вы прочитали и согласны с пользовательским соглашением http://www . mathnet.ru/rus/agreement

Параметры загрузки:

IP: 52.6 .47 .48

26 апреля 2023 г., 16:04:03

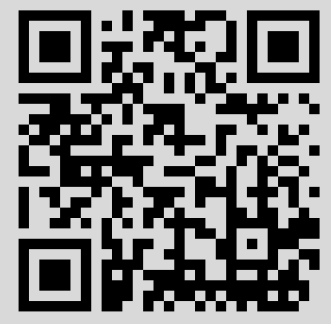




\title{
ОПИСАНИЕ СИСТЕМ КОРНЕЙ С ДАННОЙ ГРУППОЙ ВЕЙЛЯ
}

\section{И.И. Котова}

\begin{abstract}
Работа посвящена описанию обобщенных систем корней с данной группой Вейля. Эта задача возникает в связи с классификацией бесконечномерных алгебр Ли и при изучении дискретных групп, порожденных отражениями. Получен критерий существования системы корней для данной дискретной группы, порожденной отражениями, и алгоритм описания всех систем корней с данной группой Вейля.

Библиограффия: 2 названия.
\end{abstract}

1. Группы Вейля и системы корней. Настоящая работа посвящена описанию систем корней с данной группой Вейля. Эта задача возникает в связи с классификацией бесконечномерных алгебр Ли.

Для конечномерных алгебр Ли группа Вейля - это дискретная групп, порожденная отражениями, действующая в евклидовом пространстве. Существует классификация всех таких груп; каждой неприводимой дискретной групше, порожденной отражениями, соответствует не более двух систем корней. В бесконечномерном случае ситуация иная.

Пусть $A=\left(a_{i j}\right)$ - произвольная $(n \times n)$-матрица. Реализацией матрицы $A$ называется тройка $\left(\mathfrak{h}, \Pi, \Pi^{\vee}\right)$, где $\mathfrak{h}$ - векторное пространство, $\Pi=\left\{\alpha_{1}, \ldots, \alpha_{n}\right\} \subset \mathfrak{h}^{*}$, $\Pi^{\vee}=\left\{\alpha_{1}^{\vee}, \ldots, \alpha_{n}^{\vee}\right\} \subset \mathfrak{h}, \alpha_{i}\left(\alpha_{j}^{\vee}\right)=a_{i j}$. Две реализации $\left(\mathfrak{h}, \Pi, \Pi^{\vee}\right)$ и $\left(\mathfrak{h}_{1}, \Pi_{1}, \Pi_{1}^{\vee}\right)$ называются изоморфными, если сушествует изоморфизм векторных пространств $\varphi: \mathfrak{h} \rightarrow \mathfrak{h}_{1}$ такой, что $\varphi\left(\Pi^{\vee}\right)=\Pi_{1}^{\vee}$ и $\varphi^{*}\left(\Pi_{1}\right)=\Pi$.

Если наложить условия $\operatorname{dim} \mathfrak{h}=2 n-\operatorname{rk} A, \Pi$ и $\Pi^{\vee}$ линейно независимы, то реализация будет однозначно (с точностью до изоморфизма) определяться матрицей $A$. Каждая такая тройка определяет алгебру Ли, для которой $\mathfrak{h}$ является картановской подалгеброй, $\Pi$ и $\Pi^{\vee}$ являются соответственно базисом системы корней и базисом дуальной системы корней (см. [1]).

Пусть матрища $A$ обладает следующими свойствами:

а) $a_{i i}=2$ при всех $i=1, \ldots, n$;

б) $a_{i j}$ - неположительные целые числа при $i \neq j$;

в) $a_{i j}=0 \Longleftrightarrow a_{j i}=0$.

Такие матрицы назьваются обобщенными матрицами Картана, а соответствующие им алгебры Ли - алгебрами Каца-Муди.

Группа Вейля для данной матрицы $A$ с реализацией $\left(\mathfrak{h}, \Pi, \Pi^{\vee}\right)$ - это групша преобразований пространства $\mathfrak{h}^{*}$, порожденная отражениями $R_{i}$, действующими по формуле $R_{i}(x)=x-x\left(\alpha_{i}^{\vee}\right) \alpha_{i}$. 
Если $A$ является обобщенной матрицей Картана, то группа Вейля действует дискретно в некотором открытом выпуклом конусе в $\mathfrak{h}^{*}$ (см. [2]). При этом отражения $R_{1}, \ldots, R_{n}$ - это множество отражений в стенках фундаментальной камеры этой группы.

Выясним, в каком случае две матрищы задают одну и ту же группу Вейля.

Отражения $R_{i}$ и $R_{j}$ совпадают в том и только том случае, когда совпадают числа $x\left(\alpha_{i}^{\vee}\right) \alpha_{i}$ и $x\left(\alpha_{j}^{\vee}\right) \alpha_{j}$ при всех $x$ из $\mathfrak{h}^{*} ;$ это равносильно условию

$$
\alpha_{i}=\lambda \alpha_{j}, \quad \alpha_{i}^{\vee}=\frac{1}{\lambda} \alpha_{j}^{\vee} \quad(\lambda \neq 0)
$$

Таким образом, матрицы $A_{1}$ и $A_{2}$ задают одну и ту же группу Вейля в том и только том случае, когда $A_{1}$ представима в виде

$$
A_{1}=D^{-1} A_{2} D, \quad D=\operatorname{diag}\left\{\lambda_{1}, \ldots, \lambda_{n}\right\}, \quad \lambda_{i} \neq 0
$$

или $A_{1}$ получена из матрищы такого вида перестановкой индексов.

В пространстве любой размерности можно привести пример дискретной групшы, порожденной отражениями, со сколь угодно большим конечным количеством систем корней. Например, рассмотрим обобщенную матрищу Картана

$$
A=\left(\begin{array}{ccccc}
2 & -2^{m} & & & 0 \\
-2^{m} & 2 & -1 & & \\
& -1 & \ddots & \ddots & \\
& & \ddots & \ddots & -1 \\
0 & & & -1 & 2
\end{array}\right), \quad m \in \mathbb{N}
$$

порядка $n \times n$. Для нее существует реализация $\left(\mathfrak{h}, \Pi, \Pi^{\vee}\right), \operatorname{dim} \mathfrak{h}=n$, так как $A$ невырождена; $\Pi=\left\{\alpha_{1}, \ldots, \alpha_{n}\right\}, \Pi^{\vee}=\left\{\alpha_{1}^{\vee}, \ldots, \alpha_{n}^{\vee}\right\}$. При этом любая матрица

$$
A=\left(\begin{array}{ccccc}
2 & -2^{m+k} & & & 0 \\
-2^{m-k} & 2 & -1 & & \\
& -1 & \ddots & \ddots & \\
& & \ddots & \ddots & -1 \\
0 & & & -1 & 2
\end{array}\right), \quad k \in \mathbb{Z}, \quad-m \leqslant k \leqslant m
$$

с реализацией $\left(\mathfrak{h}, \Pi, \Pi^{\vee}\right)$, где

$$
\Pi=\left\{2^{k} \alpha_{1}, \alpha_{2}, \ldots, \alpha_{n}\right\}, \quad \Pi^{\vee}=\left\{2^{-k} \alpha_{1}^{\vee}, \alpha_{2}^{\vee}, \ldots, \alpha_{n}^{\vee}\right\}
$$

будет задавать ту же групу Вейля. 
2. Критерий существования системы корней с данной группой Вейля. Пусть $W$ - дискретная группа преобразований векторного пространства $\mathfrak{h}^{*}$, порожденная отражениями

$$
R_{i}(x)=x-x\left(\alpha_{i}^{\vee}\right) \alpha_{i}, \quad i=1, \ldots, n, \quad\left\{\alpha_{1}, \ldots, \alpha_{n}\right\} \subset \mathfrak{h}^{*}, \quad\left\{\alpha_{1}^{\vee}, \ldots, \alpha_{n}^{\vee}\right\} \subset \mathfrak{h} ;
$$

$(n \times n)$-матрица $A$ составлена из чисел $g_{i j}=\alpha_{i}\left(\alpha_{j}^{\vee}\right)$, причем $a_{i i}=2$ и $a_{i j}=0 \Longleftrightarrow$ $a_{j i}=0$; считаем, что $R_{1}, \ldots, R_{n}$ - это множество отражений в стенках некоторой фундаментальной камеры группы $W$.

Цель настоящей работы - предложить алгоритм, позволяющий по данной матрище $G$ описать все матрицы Картана, задающие ту же групу Вейля. Это равносильно описанию с точностью до пропорциональности всех наборов $\left\{\lambda_{1}, \ldots, \lambda_{n}\right\}, \lambda_{i} \neq 0$, таких что $A=D G D^{-1}$ - обобщенная матрица Картана, $D=\operatorname{diag}\left\{\lambda_{1}, \ldots, \lambda_{n}\right\}, \lambda_{i} \neq 0$. Другими словами, $\lambda_{i}$ должны обладать следующим свойством: $\left(\lambda_{i} / \lambda_{i}\right) \cdot g_{i j}$ - неположительные целые числа для всех $i, j=1, \ldots, n, i \neq j$.

Считаем далее, что группа $W$ неразложима, т.е. не разлагается в прямую сумму $W=W_{1} \oplus W_{2}$; это равносильно тому, что матрица $G$ путем перестановки индексов не приводится к виду

$$
G=\left(\begin{array}{cc}
G_{1} & 0 \\
0 & G_{2}
\end{array}\right) \text {. }
$$

Будем назьвать матрицы $A$ и $B$ диагонально подобными, если

$$
A=D B D^{-1}, \quad D=\operatorname{diag}\left\{\lambda_{1}, \ldots, \lambda_{n}\right\}, \quad \lambda_{i} \neq 0
$$

равенство выполнено с точностью до перестановки индексов.

ПРЕДЛОЖЕНИЕ 1. Пусть $G$ - неразложимиая $(n \times n)$-матрица, $g_{i i}=2$ u $g_{i j}=0$ $\Longleftrightarrow g_{j i}=0$. Обобщенная матрица Картана, диагонально подобная $G$, существует в том и только том случае, если все ииклические произведения

$$
g_{i_{1} i_{2}} g_{i_{2} i_{3}} \cdots g_{i_{k} i_{1}}, \quad k=1, \ldots, n,
$$

иелье, и при $i_{1} \neq i_{2}, i_{2} \neq i_{3}, \ldots, i_{k} \neq i_{1}$ знак $\operatorname{sgn}\left(g_{i_{1} i_{2}} g_{i_{2} i_{3}} \cdots g_{i_{k} i_{1}}\right)$ равен $(-1)^{k}$ или 0.

ДокАЗАТЕЛЬСтво. Необходимость. Пусть $A$-обобщенная матрица Картана, $a_{i j}=$ $\left(\lambda_{i} / \lambda_{j}\right) \cdot g_{i j}$. Тогда

$$
a_{i_{1} i_{2}} \cdots a_{i_{k} i_{1}}=\frac{\lambda_{i_{1}}}{\lambda_{i_{2}}} g_{i_{1} i_{2}} \cdots \frac{\lambda_{i_{k}}}{\lambda_{i_{1}}} g_{i_{k} i_{1}}=g_{i_{1} i_{2}} \cdots g_{i_{k} i_{1}} \in \mathbb{Z},
$$

причем $\operatorname{sgn}\left(a_{i_{1} i_{2}} \ldots a_{i_{k} i_{1}}\right)=(-1)^{k}$, если в этом циклическом произведении нет чисел $a_{i i}$.

Достаточность. В силу неразложимости матрицы $G$ для любой пары индексов $i$ и $j$, $i \neq j$, существует такая последовательность $i_{1}=i, i_{2}, \ldots, i_{k}$ такая, что $g_{i i_{2}} \cdots g_{i_{k} j} \neq 0$. Эту последовательность можно выбрать так, чтобы среди индексов $i, i_{2}, \ldots, i_{k}, j$ не было одинаковых. Обозначим

$$
\bar{g}_{i j}= \begin{cases}(-1)^{k} g_{i i_{2}} \cdots g_{i_{k} j} \neq 0 & \text { при } i \neq j, \\ 1 & \text { при } i=j\end{cases}
$$


$N_{i}$ - наибольший общий делитель циклических произведений $\left|\bar{g}_{i 1} g_{1 j_{2}} \cdots g_{j_{l} i}\right|$, не равных 0 . Хотя бы одно такое произведение существует: $g_{1 i_{2}} \cdots g_{i_{k}} g_{i i_{k}} \cdots g_{i_{2} 1}$ не равно 0 в силу условия $g_{i j}=0 \Longleftrightarrow g_{j i}=0$. Из этого неравенства также следует, что числа $\bar{g}_{i j}$ можно выбрать так, что

$$
\operatorname{sgn}\left(\bar{g}_{i j} \bar{g}_{j i}\right)=(-1)^{2 k}>0 .
$$

Пусть $\lambda_{i}=N_{i} / \bar{g}_{i j}$. Докажем, что при $D=\operatorname{diag}\left\{\lambda_{1}, \ldots, \lambda_{n}\right\}$ матрица $A=D G D^{-1}$ является обобщенной матрищей Картана. Пусть $g_{i j} \neq 0$. Тогда

$$
a_{i j}=\frac{\lambda_{i}}{\lambda_{j}} g_{i j}=\frac{\bar{g}_{j 1}}{\bar{g}_{i 1}} \frac{N_{i}}{N_{j}} g_{i j}
$$

Это число рационально, так как

$$
\frac{\bar{g}_{j 1}}{\bar{g}_{i 1}} g_{i j}=\frac{g_{i j} \bar{g}_{j 1} \bar{g}_{1 i}}{\bar{g}_{i 1} \bar{g}_{1 i}} \in \mathbb{Q} \text {. }
$$

Оно отрицательно, так как

$$
\bar{g}_{i 1} \bar{g}_{1 i}>0, \quad \operatorname{sgn}\left(g_{i j} \bar{g}_{j 1} \bar{g}_{1 i}\right)=(-1)^{k_{1}}(-1)^{k_{2}}(-1)^{k_{1}+k_{2}+1}=-1
$$

( $k_{1}$ и $k_{2}$ - это количество членов в произведениях $\bar{g}_{j 1}$ и $\bar{g}_{1 i}$ соответственно). Представим $a_{i j}$ в виде несократимой дроби $a_{i j}=m / n, m \in \mathbb{Z}, n \in \mathbb{N}$. Докажем, что $n=1$. Предположим, что $n$ делится на простое число $p$. Пусть запись $\operatorname{deg}_{p}(a)$ обозначает максимальную степень $p$, на которую делится целое число $a$. Существует циклическое произведение $g=\bar{g}_{i 1} g_{1 j_{2}} \cdots g_{j_{l}}$ такое, что $\operatorname{deg}_{p}(g)=\operatorname{deg}_{p}\left(N_{i}\right)$. Тогда при некотором целом $q$, взаимно простом с $p$,

$$
a_{i j}=\frac{1}{q} \frac{\bar{g}_{j 1} g_{i j} \bar{g}_{i 1} g_{1 j_{2}} \cdots g_{j_{l} i}}{\bar{g}_{i 1} N_{j}}=\frac{1}{q} \frac{\bar{g}_{j 1} g_{1 j_{2}} \cdots g_{j_{l} i} g_{i j}}{N_{j}} ;
$$

но число $\bar{g}_{j 1} g_{1 j_{2}} \cdots g_{j_{l}} g_{i j}$ делится на $N_{j}$. Значит, знаменатель числа $a_{i j}=m / n$ делится на $p$. Мы пришли к противоречию.

3. Описание систем корней с данной группой Вейля. Пусть дана неразложимая обобщенная матрица Картана $A$. Опишем все матрицы $D=\operatorname{diag}\left\{\lambda_{1}, \ldots, \lambda_{n}\right\}$, $\lambda_{i}>0$, такие, что $B=D A D^{-1}$ - тоже обобщенная матрица Картана. Это равносильно условию

$$
\frac{\lambda_{i}}{\lambda_{j}} a_{i j} \in \mathbb{Z} \quad \forall i, j=1, \ldots, n .
$$

Если вьполнено (1), то числа

$$
\frac{\lambda_{i}}{\lambda_{i_{1}}} a_{i i_{1}} \cdots \frac{\lambda_{i_{k}}}{\lambda_{j}} a_{i_{k} j}=\frac{\lambda_{i}}{\lambda_{j}} a_{i i_{1}} \cdots a_{i_{k} j}
$$

целые для любой последовательности индексов $i_{1}, \ldots, i_{k}$. Обозначим через $m_{i j}, i \neq j$, наибольший общий делитель произведений $\left|a_{i i_{1}} \cdots a_{i_{k} j}\right|$, не равных 0 . Такие произведения существуют в силу неразложимости $A$. Ясно, что достаточно рассматривать последовательности $i, i_{1}, \ldots i_{k}, j$ без повторений, число $m_{i j}$ от этого не изменится. Числа $m_{i j}$ 
обладают свойствами $m_{i j} \in \mathbb{N} ; m_{i j} m_{j k}$ делится на $m_{i k}$ при всех $i, j, k=1, \ldots, n$. Условие (1) равносильно следуюшему:

$$
\frac{\lambda_{i}}{\lambda_{j}} m_{i j} \in \mathbb{Z}
$$

Пусть $\lambda_{1}$ - целое число такое, что при всех $j=2, \ldots, n \lambda_{1} / m_{j 1} \in \mathbb{Z}$. Из (2) следует, что $\lambda_{j}: \lambda_{1} / m_{j 1} \in \mathbb{Z}$, поэтому $\lambda_{j}$ целые при всех $j=2, \ldots, n$.

Нам необходимо описать наборы $\left\{\lambda_{1}, \ldots, \lambda_{n}\right\}$ со свойством (2) с точностью до пропорциональности. Зафиксируем в качестве $\lambda_{1}$ целое число, которое делится на все числа $m_{j 1}, j=2, \ldots, n$. Например, возьмем $\lambda_{1}=\operatorname{HOK}\left(m_{i j}\right), i, j=2, \ldots, n$. Тогда все числа $\lambda_{j}, j=1, \ldots, n$, будут целыми.

ПРЕДЛОЖЕНИЕ 2. Пусть заданы такие числа $\lambda_{1}, \ldots, \lambda_{k}, k<n$, что

а) $\lambda_{1}$ делится на $m_{j 1}$ при всех $j=2, \ldots, k+1$;

б) условие (2) выполнено при всех $j=2, \ldots, k$.

Тогда существует $\lambda_{k+1} \in \mathbb{N}$ такое, что (2) выполнено при всех $j=2, \ldots, k+1$.

ДокАЗАТЕЛЬСТВо. Для числа $\lambda_{k+1}$ должны быть выполнены условия

$$
\frac{\lambda_{i} m_{i, k+1}}{\lambda_{k+1}} \in \mathbb{Z}, \quad \frac{\lambda_{k+1} m_{k+1, i}}{\lambda_{i}} \in \mathbb{Z} \quad \forall i=1, \ldots, k .
$$

Другими словами, $\lambda_{k+1}-$ это целое число, которое делит НОД $\left\{\lambda_{i} m_{i, k+1} \mid i=1, \ldots, k\right\}$ и делится на $\operatorname{HOK}\left\{\lambda_{i} / m_{k+1, i} \mid i=1, \ldots, k\right\}$. Такое число существует, если при всех $i, j=1, \ldots, k$ число $\lambda_{i} m_{i, k+1}$ делится на $\lambda_{j} / m_{k+1, j}$. Проверим, что это действительно так:

$$
\lambda_{i} m_{i, k+1} \frac{m_{k+1, j}}{\lambda_{j}}=\frac{\lambda_{i}}{\lambda_{j}} m_{i, k+1} m_{k+1, j}
$$

но $m_{i k+1} m_{k+1 j}$ делится на $m_{i j}$, а число $\left(\lambda_{i} / \lambda_{j}\right) \cdot m_{i j}$ целое по условию.

Мы получили, что в качестве $\lambda_{k+1}$ можно взять любое число вида

$$
\lambda_{k+1}=c \cdot \operatorname{HOK}\left\{\frac{\lambda_{i}}{m_{k+1, i}} \mid i=1, \ldots, k\right\}
$$

где $c$ делит

$$
N=\frac{\operatorname{НОД}\left\{\lambda_{i} m_{i, k+1} \mid i=1, \ldots, k\right\}}{\operatorname{HOK}\left\{\lambda_{i} / m_{k+1, i} \mid i=1, \ldots, k\right\}}=\operatorname{HОД}\left\{m_{i, k+1} m_{k+1, j} \frac{\lambda_{i}}{\lambda_{j}} \mid i, j=1, \ldots, k\right\} .
$$

Таким образом, можно предложить следующий способ построения для данной матрицы $G$ всех матрищ $D=\operatorname{diag}\left\{\lambda_{1}, \ldots, \lambda_{n}\right\}, \lambda_{i} \neq 0$, таких, что $A=D G D^{-1}$ - обобщенная матрица Картана.

Пусть матрища $G$ неразложима и удовлетворяет условиям предложения 1.

1. Построим какую-нибудь матрицу $D_{0}$, для которой $A=D_{0} G D_{0}^{-1}$ будет обобщенной матрищей Картана. Способ построения такой матрицы содержится в доказательстве предложения 1.

2. Для обобщенной матрищы Картана $A$ построим все возможные

$$
D=\operatorname{diag}\left\{\lambda_{1}, \ldots, \lambda_{n}\right\}, \quad \lambda_{i} \neq 0,
$$


при которых $D A D^{-1}$ тоже обобщенная матрица Картана. Для этого:

а) построим матрицу $M=\left(m_{i j}\right)$ : для данных $i, j=1, \ldots, n, i \neq j, m_{i j}$ - это наибольший обший делитель ненулевых произведений $\left|a_{i i_{1}} \cdots a_{i_{k}}\right|, k \geqslant 0$, где в последовательности $i_{0}=i, i_{1}, \ldots, i_{k}, j$ нет повторений;

б) зададим $\lambda_{1}=\operatorname{HOK}\left(m_{i j}\right), i, j=2, \ldots, n$ (можно взять любое другое число, делящееся на все $\left.m_{i j}\right)$;

в) предположим, что уже построены числа $\lambda_{1}, \ldots, \lambda_{k}(k<n)$, и вычислим

$$
\begin{gathered}
N_{1}=\operatorname{HOK}\left\{\frac{\lambda_{i}}{m_{k+1, i}} \mid i=1, \ldots, k\right\}, \\
N_{2}=\text { НОД }\left\{m_{i, k+1} m_{k+1, j} \frac{\lambda_{i}}{\lambda_{j}} \mid i, j=1, \ldots, k\right\} ;
\end{gathered}
$$

при данных $\lambda_{1}, \ldots, \lambda_{k}$ значение $\lambda_{k+1}$ пробегает множество чисел вида $\lambda_{k+1}=$ $c \cdot N_{1}, c \in \mathbb{N}, c$ делит $N_{2}$.

Приведенньй алгоритм (представляющий собой систему вложенных циклов) позволяет для данной обобщенной матрицы Картана перечислить все обобщенные матрищы Картана, диагонально подобные ей.

В заключение автор выражает благодарность своему научному руководителю Э.Б. Винбергу за постановку задачи и постоянное внимание к работе.

\section{СПИСОК ЦИТИРОВАННОЙ ЛИТЕРАТУРЫ}

[1] Кац В. Г. Бесконечномерные алгебры Ли. М.: Мир, 1993.

[2] Винберг Э.Б. Дискретные линейные группы, порожденные отражениями // Изв. АН CССР. Сер. матем. 1971. Т. 35. № 5. С. 1072-1112. 